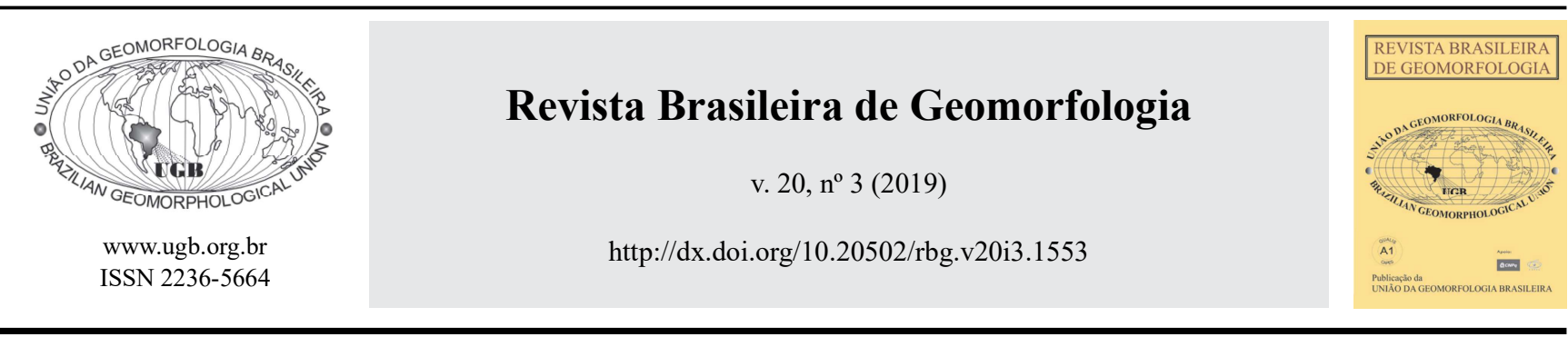

NOTA TÉCNICA

\title{
A PRODUÇÃO CIENTÍFICA EM GEOMORFOLOGIA FLUVIAL NA REVISTA BRASILEIRA DE GEOMORFOLOGIA: PANORAMA BIBLIOGRÁFICO, TENDÊNCIAS E LACUNAS
}

\section{THE SCIENTIFIC OUTPUT ON FLUVIAL GEOMORPHOLOGY IN THE REVISTA BRASILEIRA DE GEOMORFOLOGIA: BIBLIOGRAPHICAL PANORAMA, TRENDS AND GAPS}

\author{
Luiz Fernando de Paula Barros \\ Departamento de Geografia, Universidade Federal de Minas Gerais \\ Av. Antônio Carlos, 6.627. Belo Horizonte, Minas Gerais. CEP: 31270-901. Brasil \\ ORCID: http://orcid.org/0000-0001-6122-4778 \\ E-mail:luizbarros@ufmg.br \\ Rafael Augusto Pacífico dos Reis \\ Departamento de Geografia, Universidade Federal de Minas Gerais \\ Av. Antônio Carlos, 6.627. Belo Horizonte, Minas Gerais. CEP: 31270-901. Brasil \\ ORCID: http://orcid.org/0000-0001-5777-9746 \\ E-mail: rpacificoreis@gmail.com
}

\section{Informações sobre o Artigo}

Recebido (Received):

07/01/2019

Aceito (Accepted):

15/03/2019

\section{Palavras-chave:}

Geomorfologia; Produção Científica; Cursos D’água.

Keywords:

Geomorphology; Scientific Production; Watercourses.

\section{Resumo:}

Em levantamentos sobre a produção científica brasileira no século XXI, a Geomorfologia Fluvial é quase sempre apontada como o ramo da Geomorfologia com o maior número de publicações. Nesse sentido, cabe detalhar a Geomorfologia Fluvial produzida nas últimas décadas no país, contribuindo para a análise de sua evolução como ramo da ciência. Assim, o presente trabalho analisa as publicações sobre Geomorfologia Fluvial na Revista Brasileira de Geomorfologia (RBG), o único periódico nacional especializado nesta área do conhecimento. Foi feito um levantamento nas 49 edições da RBG entre os anos de 2000 e 2018 e a caracterização dos trabalhos quanto aos subtemas, autoria, instituições de origem, além de palavras-chave, recortes espaciais e abordagens técnico-metodológicas mais frequentes. Ao todo, foram analisadas 167 publicações envolvendo o estudo de sistemas fluviais e seus elementos. Os resultados mostram que ocorreu um crescimento consistente no número de publicações sobre Geomorfologia Fluvial. Há grande variedade de autores, coautores e centros de referência, com destaque para instituições federais de ensino superior das regiões sudeste e sul. A região hidrográfica do Paraná concentra o maior número de publicações, seguida pelas regiões hidrográficas do Atlântico Sudeste e São Francisco. Os principais subtemas se referem à morfologia de sistemas fluviais e/ou sua transformação, à dinâmica 
e/ou modelagem hidrossedimentológica e à morfometria de sistemas fluviais. Dentre centenas de palavras-chave utilizadas, as de maior recorrência foram "Geomorfologia Fluvial", "Neotectônica", "Luminescência Opticamente Estimulada", "Rio Paraná" e "Sensoriamento remoto". Em consonância com os dois últimos apontamentos, as abordagens técnico-metodológicas mais empregadas se referem à análise de dados topográficos, de imagens de satélite, aplicações de geoprocessamento e análise de variáveis e dados hidrossedimentológicos. Ressalta-se uma dominância de estudos com análise em escalas mais abrangentes, envolvendo bacias hidrográficas (ou um conjunto delas) e unidades de relevo. Outro recorte espacial comum é o trecho fluvial. Porém, há certa carência de estudos mais localizados, focados em sistemas de cabeceiras de drenagem e nascentes.

\begin{abstract}
:
In surveys on Brazilian scientific output in the 21 st century, fluvial geomorphology is often denoted as the branch of geomorphology with the largest number of publications. Therefore, it is important to detail the fluvial geomorphology publishing in the last two decades in the country, which contributes to the analysis of its evolution as a branch of science. Thereby, the present paper analyzes the publications on fluvial geomorphology in the Revista Brasileira de Geomorfologia (Brazilian Journal of Geomorphology; BJG), the only national journal specializing in geomorphology. A survey was conducted in the 49 editions of the BJG between 2000 and 2018. Characterization of the papers was performed according with subthemes, authors, institutions of origin, as well as more frequents keywords, spatial clippings, and technical-methodological approaches. Altogether, 167 publications were analyzed involving the study of river systems and their elements. The result shows a consistent growth in the number of publications on fluvial geomorphology. A great variety of authors, co-authors, and reference centers have been highlighted, especially federal institutions of higher education in the southeast and south regions of Brazil. The Paraná hydrographical region contains the largest number of publications, followed by the hydrographic regions of the Southeast Atlantic and São Francisco. The main subtopics refer to the morphology of fluvial systems and/ or their transformation, to the hydrosedimentological dynamics and/or its modeling, and to the morphometry of fluvial systems. Among the hundreds of keywords used, the most recurrent ones were "Fluvial Geomorphology," "Neotectonics," "Optically Stimulated Luminescence," "Paraná River," and "Remote Sensing." In agreement with the last two notes, the most used technical-methodological approaches refer to the analysis of topographic data and satellite images, applications of geoprocessing, and analysis of hydro-sedimentological aspects. It was verified a dominance of studies with large spatial scales, mainly involving watersheds and morphological units. Another common spatial cut is the river stretch. On the other hand, there was some lack of more localized studies focusing on drainage headwater systems and springs.
\end{abstract}

\section{Introdução}

A Geomorfologia se dedica ao estudo do modelado terrestre (relevo) por meio de suas formas, processos e materiais formadores. Por sua vez, a Geomorfologia Fluvial se refere ao estudo das diferentes dimensões envolvidas na estruturação espacial e na dinâmica espaço-temporal dos sistemas fluviais, além da investigação da história das paisagens fluviais (Christofoletti, 1981; Thorndycraft et al., 2008; Stevaux e Latrubesse, 2017). Assim, a Geomorfologia Fluvial abrange o estudo da complexa dinâmica presente nas interações dos cursos d'água com o espaço ao longo do tempo. Apesar de ser comumente tratada como subárea da Geografia, ela demanda (e ao mesmo tempo fornece) conhecimentos da Geologia, Biologia e engenharias, entre outras ciências, o que revela sua diversidade e amplitude. Questões relativas à dinâmica fluvial podem envolver uma grande variedade de escalas temporais - segundos a eras geológicas - e espaciais - de manchas individuais de habitats à evolução de bacias de drenagem de escala continental (Jacobson et al., 2003).

Por um lado, a Geomorfologia Fluvial se fundamenta na lógica interpretativa do uniformitarismo, que considera que "o presente é a chave do passado" (Hutton, 1788). Assim, a interpretação do passado e da história geomorfológica (em termos evolutivos) dos cursos d'água e da rede de drenagem seria embasada pelas evidências atuais, principalmente as características dos registros sedimentares. Por outro lado, uma compreensão do passado pode ser a chave para se entender o 
futuro, no sentido de que a história de um sistema fluvial pode fornecer subsídios para a compreensão das características naturais de um curso d'água e, dessa forma, servir como referencial para avaliações, restaurações e previsões (Jacobson et al., 2003), trazendo inúmeros benefícios e possibilidades para o planejamento territorial e manejo de sistemas hídricos.

No cenário científico nacional do início do século XXI, entre 2001 e 2005 e entre 2011 e 2015, a Geomorfologia Fluvial é apontada por Salgado et al. (2008) e Salgado e Limoeiro (2017), respectivamente, como a especialidade da Geomorfologia com o maior número de publicações científicas no Brasil. Entre 2006 e 2010, o tema Geomorfologia Fluvial foi superado pelo de "processos de vertente", porém foi o segundo mais trabalhado (Oliveira e Salgado, 2013). Tendo em vista esse protagonismo no âmbito da produção científica em Geomorfologia no Brasil, o presente trabalho tem como objetivo levantar e analisar o quadro da produção científica referente à Geomorfologia Fluvial na Revista Brasileira de Geomorfologia (RBG). Este é o único periódico nacional especializado nesta área do conhecimento classificado pela Coordenação de Aperfeiçoamento de Pessoal de Nível Superior (CAPES). Para tanto, foi feita uma caracterização das publicações quanto aos subtemas, autoria, instituições de origem, além de palavras-chave, recortes espaciais e abordagens técnico-metodológicas mais frequentes.

O mapeamento da produção científica da Geomorfologia Fluvial é importante para se investigar sua evolução como ramo da ciência. A caracterização e a sistematização do quadro de publicações podem contribuir para avanços nas pesquisas sobre $\mathrm{o}$ assunto, apontando-se tendências, bem como possíveis lacunas. Esta sinalização pode auxiliar, portanto, o direcionamento das pesquisas por parte de especialistas e instituições interessadas no tema. $O$ trabalho também contribui com os leitores/pesquisadores interessados em obter uma fonte integradora dos trabalhos já publicados, facilitando as consultas. Assim, como um trabalho que investiga as características da produção geomorfológica brasileira, o presente levantamento se justifica como subsídio para um maior conhecimento deste ramo da ciência no país.

\section{Procedimentos técnico-metodológicos}

Primeiramente, foi feito um levantamento nas 49 edições da RBG entre os anos de 2000 (volume 1, número 1) e 2018 (volume 19, número 4), disponíveis no sítio eletrônico da revista, a fim de selecionar os trabalhos dedicados à Geomorfologia Fluvial - conforme o entendimento exposto anteriormente. Uma vez selecionados os trabalhos, eles foram analisados e suas informações foram sistematizadas numa planilha do software Microsoft Excel. Nesta planilha foram especificadas informações como: ano de publicação; subtemas; autoria; instituições de vinculação dos autores; recortes espaciais; localização da área estudada quanto à região hidrográfica nacional, conforme a Agencia Nacional de Águas (ANA); e abordagens técnico-metodológicas.

A fim de tornar possível uma análise quantitativa de variáveis qualitativas, foram estabelecidas categorias padrão para melhor representar o universo abordado. Nestes casos, um mesmo trabalho pode estar associado a mais de uma categoria. Apesar de ser possível estabelecer outros recortes além dos definidos, optou-se por um número mais enxuto de categorias abrangentes, a fim de propiciar uma melhor visualização do conjunto dos trabalhos.

Foram definidos os seguintes subtemas para a Geomorfologia Fluvial: Dinâmica e modelagem hidrossedimentológica; Estratigrafia, reconstituição de paleoambientes e evolução do relevo; Morfologia de sistemas fluviais e/ou sua transformação; Morfometria de sistemas fluviais; e Revisões e discussões teórico-metodológicas e/ou conceituais. Em relação aos recortes espaciais, foram adotados: Bacia hidrográfica; Cabeceira de drenagem; Canal fluvial ou trecho fluvial; Conjunto de (sub)bacias hidrográficas; Estuários, deltas e planícies fluvio-marinhas; Nascente; Unidade de relevo; e Vale Fluvial. Quanto às abordagens técnico-metodológicas, foram definidas as seguintes categorias:

- Análises de dados topográficos e/ou batimétricos: análise de cartas topográficas em diferentes escalas e/ou produtos de sensores remotos (dados SRTM, ASTER, etc.), além de levantamentos batimétricos ou uso de dados secundários de batimetria;

- Análise de imagens: análise de imagens aéreas (ortofotos), de satélite (Landsat, Cbers, Quickbird, etc.) e/ou fotográficas;

- Análise de variáveis e dados hidrossedimentológicos: obtenção ou consulta a bases de dados secundários de vazão, nível da água, largura, profundidade, turbidez, etc.;

- Análises estratigráficas: descrição e interpretação 
de registros sedimentares com uso de técnicas estratigráficas diversas;

- Análises físicas e/ou químicas de amostras de água: coleta de amostras de água para mensuração de parâmetros como sólidos totais dissolvidos, $\mathrm{pH}$, concentração de elementos diversos, etc.;

- Análises geocronológicas: coleta de amostras e datação de depósitos sedimentares por técnicas diversas, como a Luminescência Opticamente Estimulada e o Radiocarbono;

- Análises granulométricas, morfológicas, químicas e/ou mineralógicas de sedimentos: coleta de amostras de sedimentos e análises em laboratório de aspectos químicos, físicos, geométricos ou de tamanho;

- Geoprocessamento: obtenção de dados e/ou cruzamento de dados em softwares, sobretudo em Sistemas de Informações Geográficas (SIG’s).

Posteriormente, foram gerados gráficos, mapa e tabelas nos softwares Microsoft Excel, QGis e Microsoft Word, a fim de evidenciar as constatações alcançadas pela análise. Cabe enfatizar que o presente trabalho não realiza avaliações quanto ao mérito das produções analisadas.

\section{Resultados e Discussões}

Ao todo, foram analisadas 167 publicações envolvendo o estudo de sistemas fluviais e seus elementos. Ressalta-se que, como o enquadramento ou não de um trabalho como sendo de Geomorfologia Fluvial é um recorte que carrega algum grau de subjetividade - tendo em vista a interpenetração de diversas áreas da geomorfologia em alguns trabalhos, optou-se por um recorte amplo, incluindo trabalhos que fornecem importantes dados e/ou análises dos sistemas fluviais, ainda que estes não sejam, necessariamente, o foco. Como exemplo, cita-se o trabalho de Marques Neto e Perez Filho (2014), sobre a "Compartimentação morfoestrutural da bacia do Rio Verde, Sul de Minas Gerais", pois, embora o foco seja a compartimentação morfoestrutural, esta revela importantes aspectos do sistema fluvial do Rio Verde em relação ao seu condicionamento geológico.

A Figura 1 traz a sistematização do número de publicações sobre Geomorfologia Fluvial por ano. Cabe ressaltar que até o ano de 2002 houve a publicação de um número da RBG por ano, passando para dois números por ano entre 2003 e 2010 (exceto 2004, quando foi publicado apenas um número). Em 2011 foram publicados três números e, a partir de então, vem sendo publicados quatro números por ano, regularmente. Em 2002 foram publicados os primeiros trabalhos afeitos à Geomorfologia Fluvial. A partir de 2011 há um crescimento significativo no número de publicações sobre o tema, atingindo um máximo de 22 trabalhos em 2018. Assim, 71,9\% dos trabalhos sobre Geomorfologia Fluvial na RBG foram publicados nos últimos 8 anos (2011 a 2018).

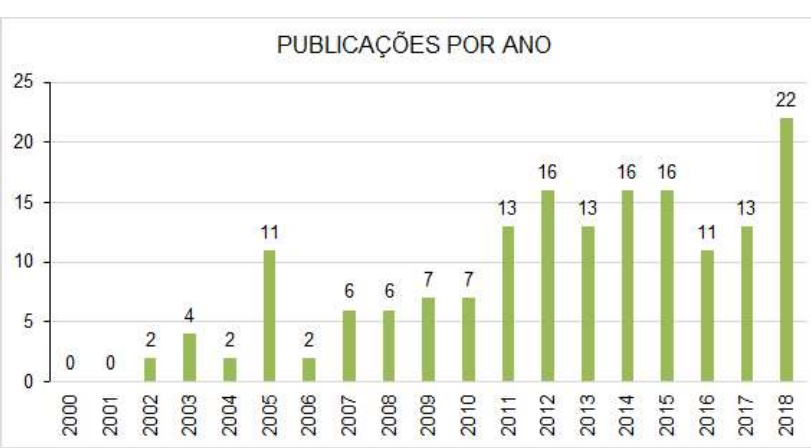

Figura 1 - Número de trabalhos sobre geomorfologia fluvial por ano de publicação na RBG.

Foram contabilizados 330 autores e coautores nas publicações analisadas. Desse total, 77,0\% participou de apenas 1 publicação. Apenas 3,3\% dos autores participou de 5 ou mais publicações (Figura 2). A análise das instituições associadas à produção científica em Geomorfologia Fluvial é semelhante, pois do total de instituições identificadas (88), 63,6\% têm apenas uma publicação associada. Por outro lado, há 11 instituições $(12,5 \%)$ com participação de pelo menos 1 pesquisador em 8 ou mais publicações (Figura 3). Ressalta-se que, exceto a Universidade Federal de Goiás e o Instituto Nacional de Pesquisas Espaciais (INPE - com diversas sedes), todas estas instituições de maior destaque estão localizadas nas regiões Sudeste e Sul do país, sendo três localizadas no estado do Paraná.

Entre as instituições identificadas, a maior parte $(43,2 \%)$ é de instituições federais de ensino superior e, secundariamente, técnico, seguidas pelas estaduais $(14,8 \%)$. Destaca-se a participação de instituições estrangeiras (12,5\%), superior à participação das instituições privadas de ensino (10,2\%). Os órgãos federais e estaduais (sobretudo, de pesquisa) estão presentes em $21,6 \%$ das publicações, com destaque para o INPE. 


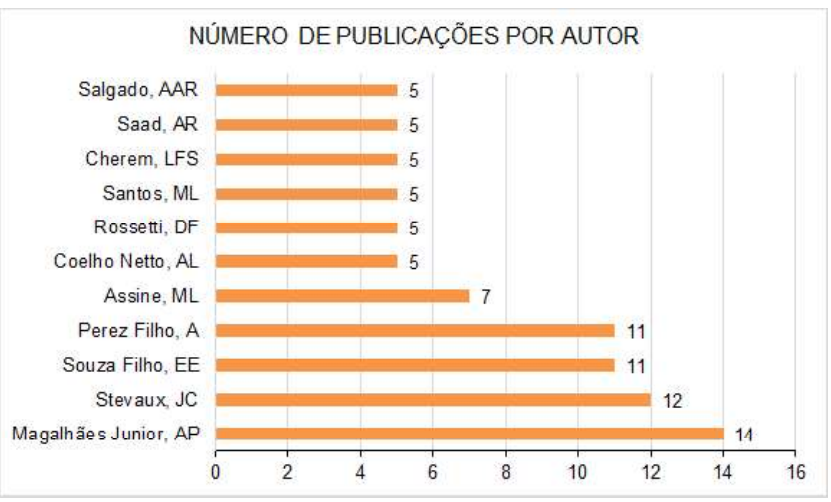

Figura 2 - Número de publicações em Geomorfologia Fluvial na RBG por autor com cinco ou mais trabalhos - uma mesma publicação pode contar com diferentes autores.

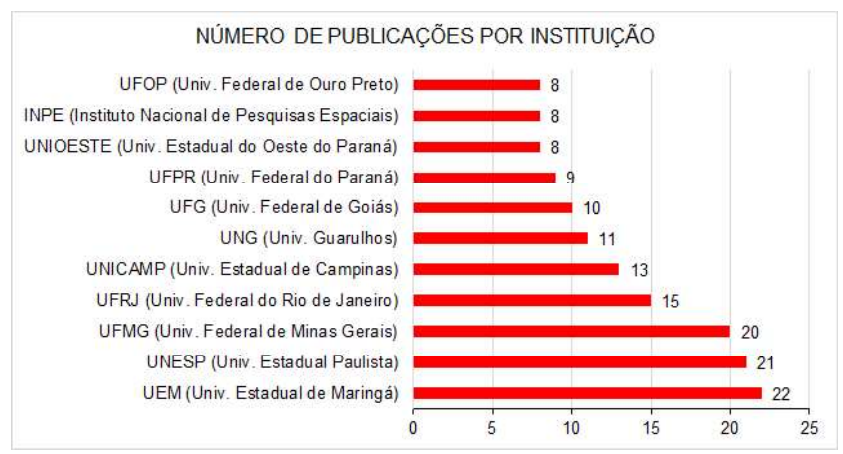

Figura 3 - úmero de publicações de Geomorfologia Fluvial na RBG por instituição de vínculo dos autores - uma mesma publicação pode contar com diferentes instituições, porém cada instituição foi contabilizada apenas uma vez em cada publicação.
Quanto às áreas mais investigadas, 155 trabalhos podem ser associados a uma das regiões hidrográficas definidas pela ANA. Assim, no mapa da Figura 4 não foram contabilizados 12 trabalhos, sendo 8 referentes a discussões teórico-metodológicas (sem estudos de casos com área definida), dois referentes a trabalhos sobre áreas fora do país e dois em zonas de divisores entre as regiões hidrográficas do Paraná, Atlântico Sudeste e São Francisco. Nota-se que a maior parte dos estudos se refere às regiões hidrográficas do Paraná (36,5\%), Atlântico Sudeste (17,4\%) e São Francisco (10,8\%), totalizando 64,7\% das publicações. Assim, nota-se certa correspondência entre a localização dos principais centros de referência com as áreas com maior número de estudos. A despeito do seu potencial hídrico, apenas $5,4 \%$ dos trabalhos estão localizados na região hidrográfica amazônica.

As palavras-chave utilizadas nos trabalhos também foram analisadas. Ao todo, foram contabilizadas 556 palavras-chave, sendo 394 diferentes. As palavras que diferenciavam apenas quanto à grafia ou à forma singular/plural foram somadas. Do total, apenas 12 palavras-chave $(3,0 \%)$ possuem 5 repetições ou mais (Tabela 1). Assim, a maior parte (81,5\%) é de palavras-chave com apenas uma ocorrência. Destacam-se as palavras-chave "Sensoriamento remoto", "Geoprocessamento" e "Luminescência Opticamente Estimulada (LOE)", pois se referem a técnicas de pesquisa de uso recorrente e crescente na Geomorfologia Fluvial.

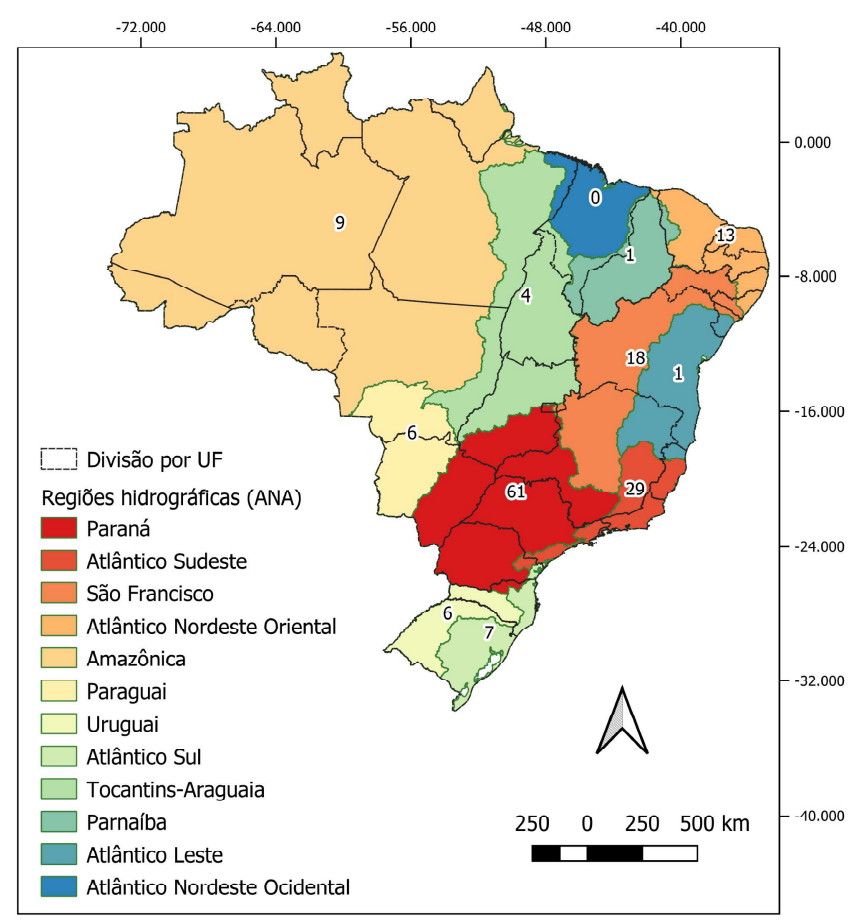

Figura 4 - Número de publicações por região hidrográfica, conforme divisão da ANA. 
Tabela 1: palavras-chave mais recorrentes nos estudos de Geomorfologia Fluvial na RBG.

\begin{tabular}{lcc}
\hline \multicolumn{1}{c}{ PALAVRA-CHAVE } & $\begin{array}{c}\text { NÚMERO DE } \\
\text { OCORRENCIAS }\end{array}$ & $\begin{array}{c}\text { \% DAS } \\
\text { PUBLICAÇÕES }\end{array}$ \\
\hline Geomorfologia Fluvial & 16 & $9,6 \%$ \\
\hline Neotectônica & 12 & $7,2 \%$ \\
\hline Luminescência Opticamente Estimulada (LOE) & 9 & $5,4 \%$ \\
\hline $\begin{array}{l}\text { Rio Paraná } \\
\text { Sensoriamento remoto }\end{array}$ & 8 & $4,8 \%$ \\
\hline Bacia Hidrográfica & 7 & $4,2 \%$ \\
\hline $\begin{array}{l}\text { Geomorfologia } \\
\text { Geoprocessamento }\end{array}$ & & \\
$\begin{array}{l}\text { Pantanal } \\
\text { Rio Ivaí }\end{array}$ & 5 & $3,0 \%$ \\
$\begin{array}{l}\text { Sistema(s) fluvial(is) } \\
\text { Uso da terra }\end{array}$ & & \\
\hline Outras & & \\
\hline & 469 & - \\
\hline
\end{tabular}

Em relação aos recortes espaciais adotados nos estudos, nota-se um domínio do uso da bacia hidrográfica - foco em 40,7\% das publicações (Figura 5). Dentre os quatro recortes mais adotados, em geral, o trecho fluvial é o de menor abrangência espacial, sendo muito comum nos estudos sobre "Dinâmica e modelagem hidrossedimentológica". Assim, estudos envolvendo recortes espaciais mais locais são menos comuns, como os que envolvem nascentes e cabeceiras de drenagem.

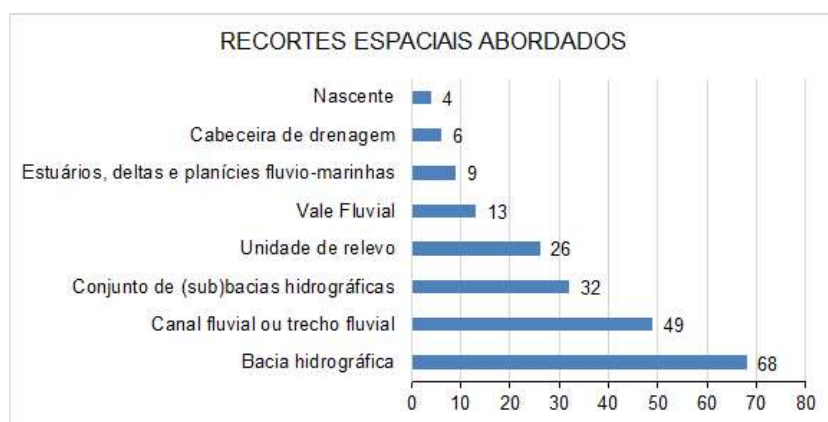

Figura 5 - Percentual de publicações por recorte espacial - um mesmo estudo pode envolver mais de um recorte.

Apesar da grande diversidade encontrada quanto aos subtemas relativos à Geomorfologia Fluvial, as categorias definidas são representativas, permitido o enquadramento dos estudos analisados (Tabela 2). Poucos trabalhos extrapolam as categorias, sobretudo aqueles de âmbito mais "ambiental" e os de "mapeamento geomorfológico-estrutural”. A caracterização morfológica de sistemas fluviais e seus elementos e/ ou sua transformação ao longo do tempo é o subtema mais recorrente (presente em $40 \%$ das publicações) e está comumente associada ao subtema "Dinâmica e modelagem hidrossedimentológica". Boa parte desses estudos lança mão da análise imagens multitemporais como abordagem técnico-metodológica, uma das mais empregadas no universo analisado (Tabela 3 ) - presente em $51 \%$ das publicações.

Em relação às abordagens técnico-metodológicas, destaca-se ainda a presença marcante do emprego de análises de dados topográficos e/ou batimétricos (57\% das publicações), sobretudo imagens da Shuttle Radar Topography Mission (SRTM). Frequentemente, essa abordagem foi utilizada em conjunto com as análises de imagens e aplicações de geoprocessamento, como comumente verificado em trabalhos sobre morfometria de sistemas fluviais. Outro procedimento comum, porém não contabilizado, foi o emprego de análises estatísticas, sobretudo em trabalhos a respeito de dinâmica e/ou modelagem hidrossedimentológica.

\section{Considerações finais}

A análise da produção científica em Geomorfologia Fluvial na Revista Brasileira de Geomorfologia 
Tabela 2: subtemas mais investigados nos estudos de Geomorfologia Fluvial na RBG - um mesmo estudo pode envolver mais de subtema.

\begin{tabular}{lcc}
\hline \multicolumn{1}{c}{ SUBTEMAS } & $\begin{array}{c}\text { NÚMERO DE } \\
\text { OCORRÊNCIAS }\end{array}$ & $\begin{array}{c}\text { \% DAS } \\
\text { PUBLICAÇÕES }\end{array}$ \\
\hline Morfologia de sistemas fluviais e/ou sua transformação & 67 & $40 \%$ \\
Dinâmica e/ou modelagem hidrossedimentológica & 58 & $35 \%$ \\
Morfometria de sistemas fluviais & 43 & $26 \%$ \\
$\begin{array}{l}\text { Estratigrafia, reconstituição de paleoambientes e evolução } \\
\text { do relevo }\end{array}$ & 27 & $16 \%$ \\
$\begin{array}{l}\text { Revisões e discussões teórico-metodológicas e/ou } \\
\text { conceituais }\end{array}$ & 15 & $9 \%$ \\
\hline
\end{tabular}

Tabela 3: abordagens técnico-metodológicas mais empregadas nos estudos de Geomorfologia Fluvial na RBG - um mesmo estudo pode envolver mais de uma abordagem.

\begin{tabular}{lcc}
\hline \multicolumn{1}{c}{ ABORDAGENS TÉCNICO-METODOLÓGICAS } & $\begin{array}{c}\text { NÚMERO DE } \\
\text { OCORRÊNCIAS }\end{array}$ & $\begin{array}{c}\text { \% DAS } \\
\text { PUBLICAÇÕES }\end{array}$ \\
\hline Análises de dados topográficos e/ou batimétricos & 96 & $57 \%$ \\
Análise de imagens (aéreas, de satélite, fotográficas) & 86 & $51 \%$ \\
Geoprocessamento & 70 & $42 \%$ \\
Análise de variáveis e dados hidrossedimentológicos & 46 & $28 \%$ \\
Análises estratigráficas & 29 & $17 \%$ \\
Análises granulométricas, morfológicas, químicas e/ou & 27 & $16 \%$ \\
mineralógicas de sedimentos & & \\
Análises geocronológicas & 17 & $10 \%$ \\
Análises físicas e/ou químicas de amostras de água & 13 & $8 \%$ \\
\hline
\end{tabular}

permitiu verificar um crescimento significativo desta área do conhecimento ao longo das 49 edições da revista, tendo ocorrido um pico de publicações sobre o tema em seu último número avaliado (v. 19, n. 4, de 2018). Isso pode ser considerado um reflexo da crescente valorização dos sistemas hídricos sob uma perspectiva de cenários de escassez hídrica num futuro próximo em diversas regiões. Além disso, a água em movimento é, sem dúvida, o principal agente atuante na esculturação do relevo, sobretudo em regiões de climas úmidos como os preponderantes no Brasil. Assim, uma análise geomorfológica completa e aprofundada frequentemente passa pela abordagem dos sistemas fluviais, suas formas, sua dinâmica, seus processos e materiais associados.

Entretanto, o entendimento dos sistemas fluviais brasileiros é ainda bastante desigual, havendo forte concentração de estudos nas regiões hidrográficas do Paraná e Atlântico Sudeste, onde também se concentram as principais instituições envolvidas na produção de conhecimento sobre o tema. Regiões hidrográficas como as do Atlântico Leste, Atlântico Nordeste Ocidental e Parnaíba são ainda praticamente vazios de dados, além de haver um enorme potencial de estudos na região Amazônica.

Observa-se um crescente uso de técnicas associadas a produtos de sensoriamento remoto e aplicações de geoprocessamento, permitindo a execução de estudos a baixos custos. O uso de métodos diretos de datação também tem sido crescente, com um forte destaque para a Luminescência Opticamente Estimulada, em detrimento de técnicas tradicionais como o radiocarbono. A inovação técnico-metodológica é essencial para a manutenção e o desenvolvimento da Geomorfologia Fluvial. Porém, é preciso estar atento à supremacia dos dados e evidências de campo, nem sempre presentes ou devidamente explorados em trabalhos baseados em dados morfométricos obtidos indiretamente ou em dados de laboratório. 


\section{Agradecimentos}

Os autores agradecem ao Conselho Nacional de Desenvolvimento Científico e Tecnológico (CNPq) pelo financiamento do projeto de pesquisa e bolsa de iniciação científica.

\section{Referências Bibliográficas}

CHRISTOFOLETTI, A. Geomorfologia fluvial. São Paulo: Edgar Blucher, 1981. 313 p.

HUTTON, J. Theory of the Earth. Transactions of the Royal Society of Edinburgh, v. 1, p. 209-304, 1788.

JACOBSON, R.; O'CONNOR, J.E.; OGUCHI, T. (2003). Surficial geologic tools in fluvial geomorphology. In KONDOLF, G. M.; PIEGAY, H. (Ed.). Tools in fluvial geomorphology. Wiley, 2003, Nova Iorque: p. 25-57.

MARQUES NETO, R.; PEREZ FILHO, A. Compartimentação morfoestrutural da bacia do Rio Verde, Sul de Minas Gerais. Revista Brasileira de Geomorfologia, v. 15, n. 1, p. 119-135, 2014. DOI: $10.20502 /$ rbg.v15i1.478
OLIVEIRA, C. K. R.; SALGADO, A. A. R. Geomorfologia Brasileira: Panorama Geral da Produção Nacional de Alto Impacto no Quinquênio entre 2006-2010. Revista Brasileira de Geomorfologia, v. 14, n. 1, p. 117-123, 2013. DOI: 10.20502/ rbg.v14i1.421

SALGADO, A. A. R; BIAZINI, J.; HENNIG, S. Geomorfologia Brasileira: Panorama Geral da Produção Nacional no Início do Século XXI (2001-2005). Revista Brasileira de Geomorfologia, v. 9, n. 1, p. 85-91, 2008. DOI: 10.20502/rbg.v9i1.104

SALGADO, A. A. R; LIMOEIRO, B. F. Geomorfologia Brasileira: Panorama Geral da Produção Nacional de Alto Impacto no Quinquênio entre 2011-2015. Revista Brasileira de Geomorfologia, v. 18, n. 1, p. 225-236, 2017. DOI: 10.20502/ rbg.v18i1.1154

STEVAUX, J. C.; LATRUBESSE, E. M. Geomorfologia fluvial. São Paulo: Oficina de Textos, 2017. 336p.

THORNDYCRAFT, V. R.; BENITO, G.; GREGORY, K. J. Fluvial geomorphology: a perspective on current status and methods. Geomorphology, v. 98, p. 2-12, 2008. DOI: 10.1016/j. geomorph.2007.02.023 